\section{Priming for chemotherapy}

Chemotherapy has been used to treat cancer for 60 years, despite an incomplete understanding of the mechanisms by which it is more toxic to cancer cells than normal cells. Now, Anthony Letai and colleagues show that a property called mitochondrial priming correlates with the clinical response to chemotherapy (Science, published online 27 October 2011; doi:10.1126/ science.1206727). Chemotherapeutic drugs induce apoptosis or inactivate anti-apoptotic pathways. To measure a tumor cell's propensity to undergo mitochondrially mediated apoptosis, the authors exposed cells to $\mathrm{BH} 3$ peptides from pro-apoptotic proteins and monitored mitochondrial depolarization. The authors termed this property, which essentially measures the proximity of mitochondria to the apoptotic threshold, 'mitochondrial priming'. They then analyzed mitochondrial priming in 85 tumors before treatment. For each tumor type, the authors found evidence supporting a correlation between higher mitochondrial priming and a positive clinical response to chemotherapy. They tested whether modulating priming would alter chemosensitivity in vitro. Using ABT-737, a BH3-mimetic drug, to increase priming in a myeloid leukemia cell line, they observed an increase in sensitivity to three chemotherapeutic agents. The authors suggest that agents that selectively increase mitochondrial priming in cancer cells may enhance the clinical response to conventional chemotherapy.

\section{PTEN ceRNAs in melanoma}

Pier Paolo Pandolfi and colleagues recently hypothesized that proteincoding RNA transcripts can compete for and sequester microRNAs (miRNAs), and they call these RNA transcripts competing endogenous RNAs (ceRNAs). In two recent studies, Pandolfi and colleagues identify ceRNAs that regulate the tumor suppressor PTEN and can promote melanoma in a mouse model (Cell 147, 344-357, 2011, and Cell 147, 382-395, 2011). The authors performed a Sleeping Beauty transposon screen to search for genes that promote melanoma in a B-RAF ${ }^{\mathrm{V} 619 \mathrm{E}}$ mouse. They identified 320 common insertion sites and searched for genes enriched for miRNA recognition elements (MREs) shared with PTEN. They identified 33 putative ceRNAs affecting PTEN and depleted them using RNA interference in human melanoma cells; the decreased levels of six of the putative ceRNAs reduced PTEN mRNA levels. None of the putative PTEN ceRNAs encode known tumor suppressors, but one gene identified, ZEB2, encodes an activator of the epithelial-to-mesenchymal transition (EMT). To test whether the regulation of PTEN by ZEB2 depends on miRNA, the authors depleted ZEB2 in a Dicer-null cell line. They found that ZEB2 knockdown did not affect $P T E N \mathrm{mRNA}$ levels, indicating that $Z E B 2$ regulation of PTEN does depend on miRNA. Using bioinformatic analysis and RNA immunoprecipitations, the authors show that the ZEB2 transcript binds at least four miRNAs in the regulation of PTEN expression. PF

\section{Personalized asthma control}

About a third of individuals with asthma who use inhaled glucocorticoids do not respond to this form of anti-inflammatory asthma control. Heterogeneity in the levels of endogenous glucocorticoids partially explains the observed variation, but family studies also suggest heritable components in the differential response to glucocorticoids. A new study reports that the promoter region of GLCCI1 harbors two linked SNP markers associated with glucocorticoid response (N. Engl. J. Med. 365,

Written by Orli Bahcall, Pamela Feliciano, Wayne Peng, \& Kyle Vogan
1173-1183, 2011). The discovery was made using a family-based genomewide screen in 403 child-parent trios, including 118 affected children who received budesonide, and was subsequently validated in four trial data sets. Individuals homozygous for the risk alleles have a 2.36-fold greater chance of poor response to inhaled glucocorticoids, and their mean improvement in a standard lung function measurement is only $30 \%$ of that of people with wild-type alleles. Reporter assays showed that the risk haplotype reduces GLCCI1 transcription; however, the transcription factors involved and the function of the GLCCI1 protein remain unknown. Together with a recent report of a phase 2 trial for lebrikizumab, in which serum periostin level accurately predicted efficacy (N. Engl. J. Med. 365, 1088-1098, 2011), this study brings us closer to personalized asthma treatment.

WP

\section{AKT2 mutations and hypoglycemia}

Loss of AKT2 function in humans causes severe insulin resistance. A new study by Robert Semple and colleagues (Science 334, 474, 2011) now shows that gain-of-function AKT2 mutations result in severe hypoglycemia, weight gain and gross asymmetric overgrowth. The authors performed exome sequencing on a single individual with these phenotypic features and identified a heterozygous missense mutation in AKT2 that was absent from the unaffected parents. They then sequenced two additional unrelated individuals with the same constellation of features and found the identical AKT2 mutation in both individuals, of whom one was heterozygous for the mutation and one showed evidence of somatic mosaicism. In both instances, the mutation was absent from the unaffected parents. The mutation, which leads to a glutamate-to-lysine substitution at position 17 in the pleckstrin homology domain of AKT2, results in constitutive plasma membrane localization and activated signaling. Notably, somatic mosaicism for the AKT1 variant encoding an identical p.Glu17Lys substitution was recently shown to underlie the characteristic skin and skeletal overgrowth in Proteus syndrome (N. Engl. J. Med. 365, 611-619, 2011), highlighting the overlapping but distinct consequences of deregulated AKT1 and AKT2 signaling.

$K V$

\section{Leishmaniasis genomes}

Visceral leishmaniasis, the most severe and potentially fatal form of leishmaniasis, is caused by the Leishmania donovani species complex. Matthew Berriman and colleagues report a high-quality reference genome sequence for a $L$. donovani strain from Nepal (Genome Res. published online, 28 October 2011; doi:10.1101/ gr.123430.111). They also isolated and sequenced the genomes of 16 clinical strains from individuals in the same region with visceral leishmaniasis. The whole-genome sequence data set provides evidence of population structure beyond that detected using traditional multilocus typing, and there is evidence of adaptive evolution, including selection on genes with surface- and transport-related functions. Drug resistance was seen to emerge independently several times in the strains infecting this group of patients. In an accompanying paper, Jeremy Mottram and colleagues report a reference genome for $L$. mexicana and refine the three other Leishmania reference genomes (Genome Res. published online, 28 October 2011; doi:10.1101/gr.122945.111). Using comparative analyses, the authors identify a small number of genes and paralog groups unique to each of these species. They find high levels of gene copy-number variation between the species implicated in leishmaniasis. They also find that aneuploidy arises frequently, resulting in chromosome copy-number variation between leishmaniasis strains and species. 